\title{
Der Nationalpark Picos de Europa - grüne Oase im Norden Spaniens
}

\author{
Hilke Steinecke \& Peter Schubert
}

\begin{abstract}
The Picos de Europa is a range of mountains near the northern coast of Spain. The national parc Picos de Europa was founded in 1995. The highest peak has an altidtude of $2650 \mathrm{~m}$. Rainfall is high. Landscape is characterized by large beech and oak forests. Vegetation zones and their typical plants are outlined.
\end{abstract}

\section{Zusammenfassung}

Die Picos de Europa sind ein Gebirge an der Nordküste Spaniens. Der gleichnamige Nationalpark wurde 1995 gegründet. Der höchste Gipfel erreicht 2650 m. Da es sehr viel regnet, findet man hier ausgedehnte Buchen- und Eichenwälder. Die verschiedenen Höhenstufen mit ihrer typischen Pflanzenwelt werden vorgestellt.

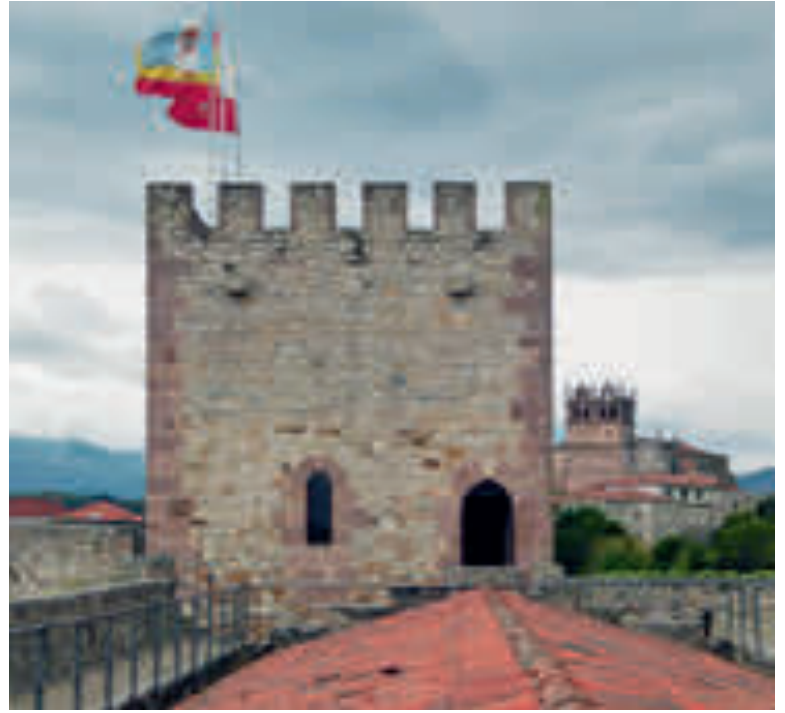

\section{Einleitung}

Mit Spanien verbinden viele eine zumindest im Sommer heiße und trockene Landschaft mit mediterranen Hartlaubgewächsen. Dieses Klischee passt aber längst nicht zu allen Regionen Spaniens. Denn gerade im Norden in der Nähe der Atlantikküste bringen feuchte Luftmassen aus Nordwesten viel Regen mit sich. Dieser sorgt dafür, dass sich eine üppige, grüne Vegetation mit ausgedehnten sommergrünen Laubwäldern entwickeln kann. Die Picos de Europa sind extrem atlantisch geprägt, weil küstennah steile Berge aufragen, an denen sich die Wolken regelmäßig und besonders stark abregnen.

Die Picos de Europa erheben sich wie eine westliche Verlängerung der Pyrenäen an der Nordküste Spaniens. Der jährliche durchschnittliche Niederschlag liegt bei $2000 \mathrm{~mm}$, was über $3 \mathrm{Mal}$ mehr als in Frankfurt ist. Niederschläge fallen hier über das Jahr relativ gleichmäßig verteilt mit Maxima von November bis Januar, in den Sommermonaten Juni bis August ist es etwas trockener. Häufig ist es wolkig oder nebelig. Als Wanderer muss man selbst im Mai noch mit schlechtem Wetter rechnen, sodass äußerste Vorsicht und gute Beobachtung des Wetters bei der Planung einer Bergtour gefordert sind. Wenn man mit dem Flugzeug via Santander anreist, sind die Picos leicht und schnell erreichbar.

\section{Der Nationalpark}

Für Natur-, Pflanzen- und Wanderfreunde sind die Picos de Europa trotz des unsicheren Wetters ein ideales Reiseziel. Vor allem wenn es im Sommer im Süden der Iberischen Halbinsel unerträglich heiß ist, zieht es viele Spanier in die kühle Sommerfrische dieses Kantabrischen Küstengebirges. So ist der Nationalpark Picos de Europa auch einer der meistbesuchten Nationalparks Spaniens. Er liegt nur rund $20 \mathrm{~km}$ von der Küste entfernt und befindet sich in den Provinzen Asturien, Kantabrien und KastilienLeón. Auf einer relativ kleinen Fläche erheben sich rund 200 Gipfel, die über $2000 \mathrm{~m}$ hoch sind. Die höchsten Gipfel gibt es im Zentralmassiv (Torre de Cerredo, $2648 \mathrm{~m}$ ). Weit vom Meer aus sind die bis in den Sommer schneebedeckten Gipfel erkennbar, es gibt hier sogar wenige kleine Gletscher. Für Seeleute, die nach einer Atlantiküberquerung von Amerika zurückkehrten, waren die Gipfel früher das erste 
Zeichen dafür, wieder zurück in Europa zu sein. Die Berge galten als natürliche Leuchttürme und wurden deshalb Picos de Europa genannt. Aber auch für Pilger und Wanderer, die den am Fuße der Picos verlaufenden Jacobsweg bis nach Santiago de Compostela ablaufen, ist das Gebirge ein markantes Landschaftselement. Pittoresk liegt das von vielen Touristen historische mittelalterliche Städtchen San Vicente de la Barquera direkt am Meer, während sich im Hintergrund die steilen Berge erheben.

Das waldreiche Hochgebirge beherbergt eine ganze Reihe besonderer Pflanzen und seltener Tiere wie Bären, Wölfe, Wildkatzen, PyrenäenGämse, Steinadler, Auerhahn oder Gänsegeier. Teile des Gebirges wurden deshalb bereits 1918 unter Schutz gestellt (Nationalpark Covadonga, erster Nationalpark Spaniens). Mit der Gründung des Nationalparks Picos de Europa am 30. Mai 1995 erfolgte eine flächenmäßige Erweiterung des Schutzes nun auch in den östlichen Teilen des Kantabrischen Gebirges. Die Picos de Europa sind heute Spaniens größter Nationalpark mit einer west-östlichen Ausdehnung von $40 \mathrm{~km}$ bei einer Breite von $20 \mathrm{~km}$ in NordSüd-Richtung. Seit 2003 hat er zudem den Status eines UNESCO-Biosphärenreservates. Ist man als Urlauber mit dem Auto unterwegs, sind die Entfernungen zwischen zwei Zielen nicht zu unterschätzen, da wegen der großen Schluchten die Straßen oft große Umwege machen.

Die Entstehung der Picos de Europa geht auf das Karbon zurück. Das Gebirge hat sich aufgefaltet, weil Iberische Halbinsel und afrikanische Kontinentalplatte aufeinanderstoßen. Das Kantabrische Gebirge gliedert sich in ein westliches (Cornión), zentrales (Urrieles) und östliches Massiv (Ándara). Besonders im östlichen und zentralen Massiv steht kompakter Kalkstein an. Oberflächlich kommt es aber stellen-

Abb. 1 (Seite 96): Der alte Hafenort San Vicente de la Barquera, im Hintergrund die Picos de Europa.

Abb. 2 (oben): In den Bergdörfern der nicht zu hohen Lagen gedeihen sogar Palmen.

Abb. 3 (unten): Die Picos de Europa vom Atlantik aus betrachtet.
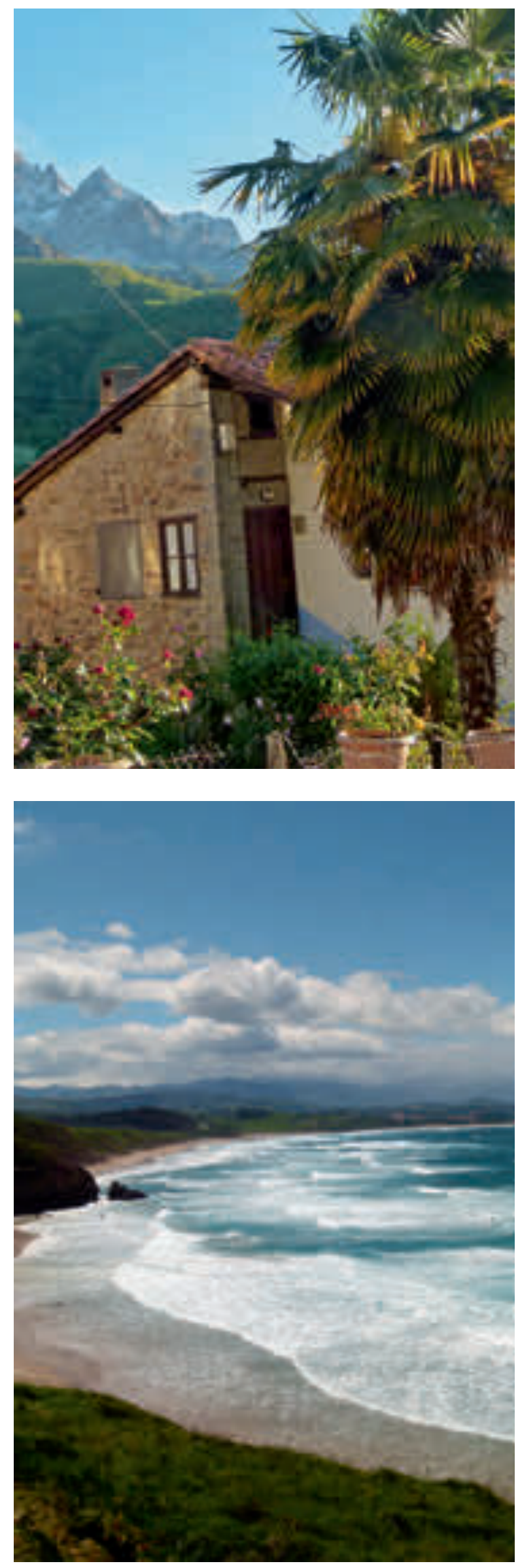

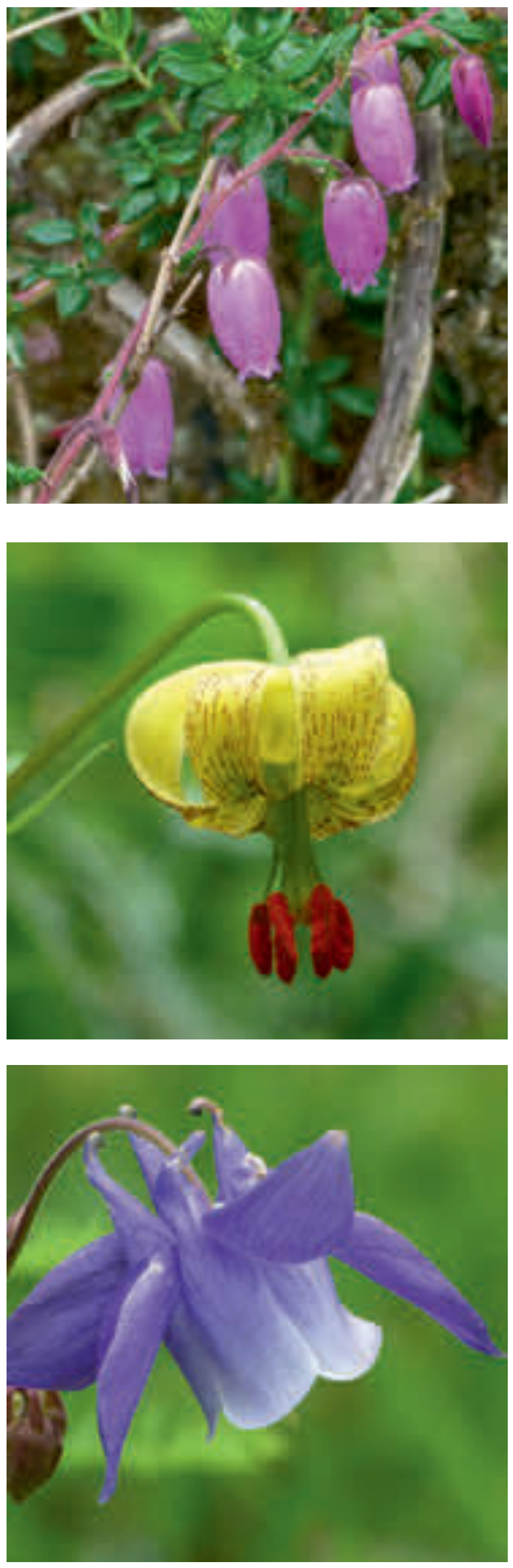

weise zu Versauerungen, sodass auch Säugezeiger wie Daboecia cantabrica und andere Heidekrautgewächse auftreten. An vielen Stellen gibt es eindrucksvolle Karsterscheinungen mit Dolinen, unterirdischen Bächen (die plötzlich wieder aus dem Fels austreten) und unzähligen Höhlen. Eine der faszinierendsten und für Besucher gut erschlossenen Höhlen am Fuße der Picos ist die Tropfsteinhöhle El Soplao. Einmalig in ihr sind die verzweigten Tropfsteine, die aussehen, als seien sie von Eiskristallen überzogen. Speleologen aus der ganzen Welt besuchen die Höhle, um dieses Phänomen zu erforschen. In den Picos de Europa liegen einige der tiefsten Höhlen der Welt, darunter Torca del Cerro (-1589 m), Sima de la Cornisa (-1507 m), Torca los Rebecos (-1255 m) und Pozo del Madejuno (-1252 m). Durch die hohen Niederschläge führen die Bergflüsse reichlich Wasser und haben im Laufe der Zeit beeindruckend gewaltige Schluchten eingeschnitten (Menendez de La Hoz o. J.)

Die schroffen Kalkfelsen verwittern leicht, an manchen Stellen erinnern die Berge etwas an die Dolomiten. Einer der markantesten Gipfel des Gebietes ist der $2518 \mathrm{~m}$ hohe Naranjo de Bulnes (Orange von Bulnes, auch asturisch Pico Urriello genannt), der bei tief stehender Sonne rotorange leuchtet. In die Höhen des Macizo Central kann man zu Fuß aufsteigen oder mit der Seilbahn von Fuente Dé bequem hochfahren. Zentrum des Tourismus ist der beschauliche alte Ort Potes. Über den Nationalpark kann man sich im nahegelegenen Infozentrum in Gangas de Onis erkundigen.

\section{Höhenstufen}

\subsection{Colline und montane Stufe}

Aufgrund des ausgeprägten Reliefs und der großen Höhenunterschiede lassen sich deutliche Höhenstufen abgrenzen. Die colline Stufe erreicht 800 Höhenmeter. Hier wird Landwirtschaft betrieben. Neben der immergrünen Steineiche kommen in den Wäldern auch Eschen, Sommer-Linden, Vogel-Kirschen und Ulmen vor. Die montane Stufe reicht bis 1800 m hinauf. Hier gibt es ausgedehnte Wälder, in denen verschiedene Eichen-Arten wach- 
sen, darunter Stiel-, Trauben-, Pflaum- und Pyrenäen-Eiche. Die Bäume sind extremen Witterungsbedingungen wie starken Winden und schweren Gewittern ausgesetzt. Dementsprechend sind viele von ihnen knorrig, schief und krumm gewachsen und häufig durch Blitzeinschläge innen hohl und verkohlt. Da sich ihr Holz nicht als Bauholz verwenden lässt, die Eicheln dagegen für die Viehmast geeignet sind, sind die Bäume von der Abholzung verschont geblieben. In den Wäldern gibt es noch reichlich stattliche, bizarre Eichen-Veteranen mit Stammdurchmessern von durchaus $2 \mathrm{~m}$ bei einem geschätzten Alter von mindestens 500 Jahren. Die Zweige der Eichen sind häufig von Flechten überwachsen, ein Hinweis auf die hohe Luftfeuchtigkeit. In den höheren Lagen werden die Eichen durch Rotbuchen ersetzt. Weitere Gehölze dieser Wälder sind Vogelbeeren, Mehlbeeren, Haselnüsse, Moor-Birken, Eiben und Stechpalmen. Auf den Reichtum an Stechpalmen (Ilex aquifolium) nimmt der Name der Siedlung Las Ilces Bezug. Die 1000-jährige Eibe an der alten Kirche Santa Maria de Lebena wurde leider im Jahr 2007 durch einen Sturm zerstört, an sie erinnert nur noch eine Infotafel sowie der Rest des toten Baumstammes. In der Waldzone werden seit Jahrhunderten Ess-Kastanien angepflanzt. Es sind bis heute alte Kastanienhaine erhalten (z. B. in der Nähe von Pineres auf den Bergen östlich des Flusses Deva) mit knorrigen, dicken Bäumen, die vermutlich aus dem 16. Jh. stammen. Sie sind bisweilen ziemlich hohl, dennoch ist noch so viel Lebenskraft in ihnen, dass sie von unten immer wieder austreiben, dadurch um so verwunschener aussehen. Die alten Bäume produzieren noch immer reichlich Früchte.

Abb. 4 (Seite 98 oben): Daboecia cantabrica.

Abb. 5 (Seite 98 Mitte): Lilium pyrenaicum.

Abb. 6 (Seite 98 unten): Aquilegia pyrenaica subsp. discolor.

Abb. 7 (oben): Alter Eichenhain mit Trauben-Eichen.

Abb. 8 (unten): Etwa 500 Jahre alte,

hohle Castanea sativa.
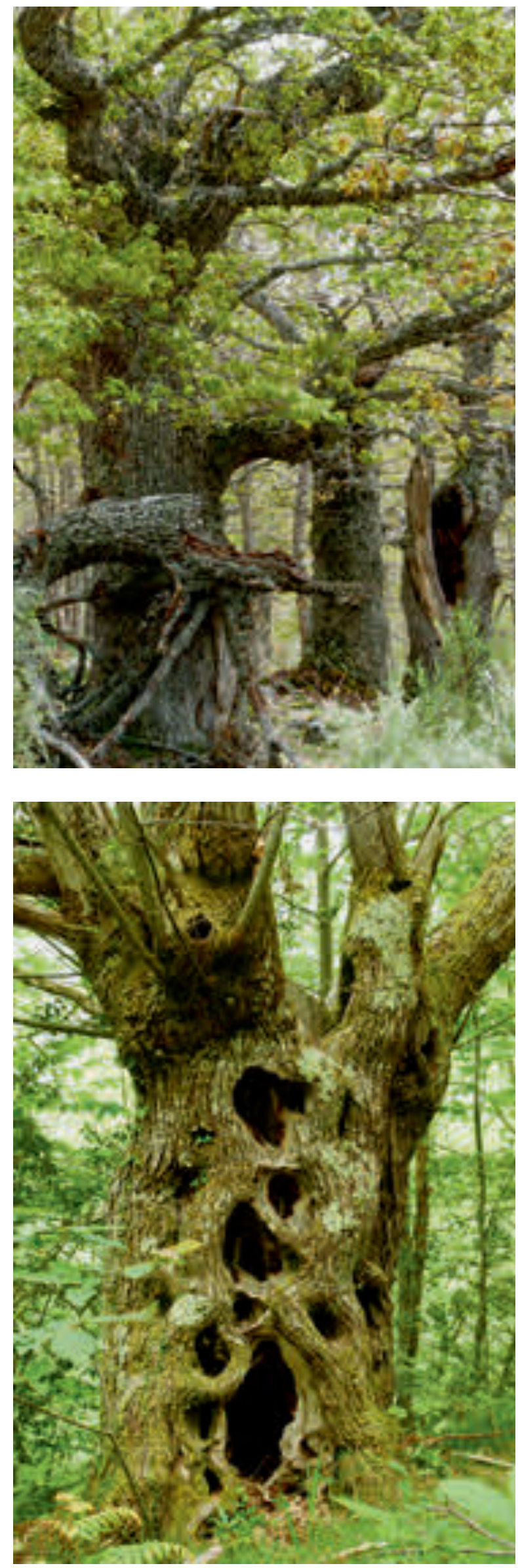

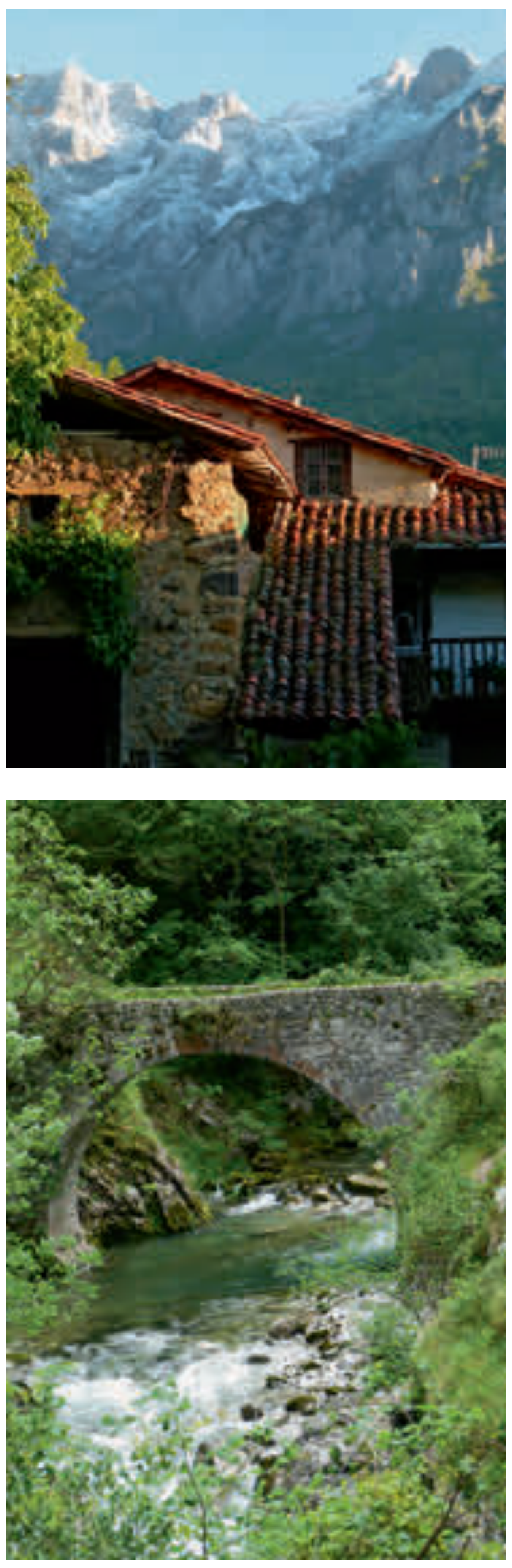

Am Waldrand oder auf Lichtungen sind viele Arten anzutreffen, die es auch in den Pyrenäen gibt, wie bereits der Artbeiname vermuten lässt. Im Mai fällt beispielsweise die Pyrenäen-Akelei (Aquilegia pyrenaica subsp. discolor) mit ihren leuchtend blauen, innen etwas helleren Blüten auf. Die Unterart ist ein Endemit der Kantabrischen Kordillere. Ein Vertrauter aus unseren Wäldern ist das Leberblümchen (Hepatica nobilis), wobei hier die Blüten kleiner als bei uns und weiß gefärbt sind. In den Wäldern, aber auch bis über die Baumgrenze bis $2000 \mathrm{~m}$ Höhe wächst auf Kalkgestein der Pyrenäen-Gamander (Teucrium pyrenaicum). In den feuchten Wäldern entdeckt man mit etwas Glück die Verborgene Schuppenwurz (Lathraea clandestina). Der Vollparasit wird wie die Sommerwurz zu den Orobanchaceae gestellt. Das Verbreitungsgebiet erstreckt sich vom nördlichen Spanien über Frankreich, das westliche von Belgien bis nach Mittelitalien. In Deutschland ist sie winterhart und wird gelegentlich in Gärten gepflanzt, wo sie gut z. B. unter Pappeln gedeiht. Im Mai blühen noch die letzten Frühjahrsgeophyten. Häufig sind Frühlings-Blaustern (Scilla verna) mit blassblauen Blüten und das in unseren Gärten auch beliebte Hasenglöckchen ( $H y$ acinthoides hispanica) anzutreffen. In Nordspanien sowie Süd- und Zentralfrankreich ist der Pyrenäen-Blaustern (Scilla lilio-hyacinthus) heimisch. Er kommt in Buchenwäldern zwischen 600 und 1600 Meter Höhe vor. Das Zwiebelgewächs fällt durch seine kräftigen Blätter und Blütenstände auf und wird auch als Zierpflanze in Gärten kultiviert. Seltener dagegen ist die Pyrenäen-Lilie (Lilium pyrenaicum), die bevorzugt auf feuchten, humusreichen, nicht nassen Böden bis auf $1800 \mathrm{~m}$ vorkommt. Sie wird bis 1,20 $\mathrm{m}$ hoch und ähnelt der Türkenbund-Lilie. Ihre Blüten sind aber gelb gefärbt. Bis auf

Abb. 9 (oben): Im alten Bergdorf Redo, im Hintergrund die schroffen Gipfel.

Abb. 10 (unten): Alte Steinbrücke über den Rio Urdón.

Abb. 11 (Seite 101): Von Genista hispanica gelb gefärbte Berge. 


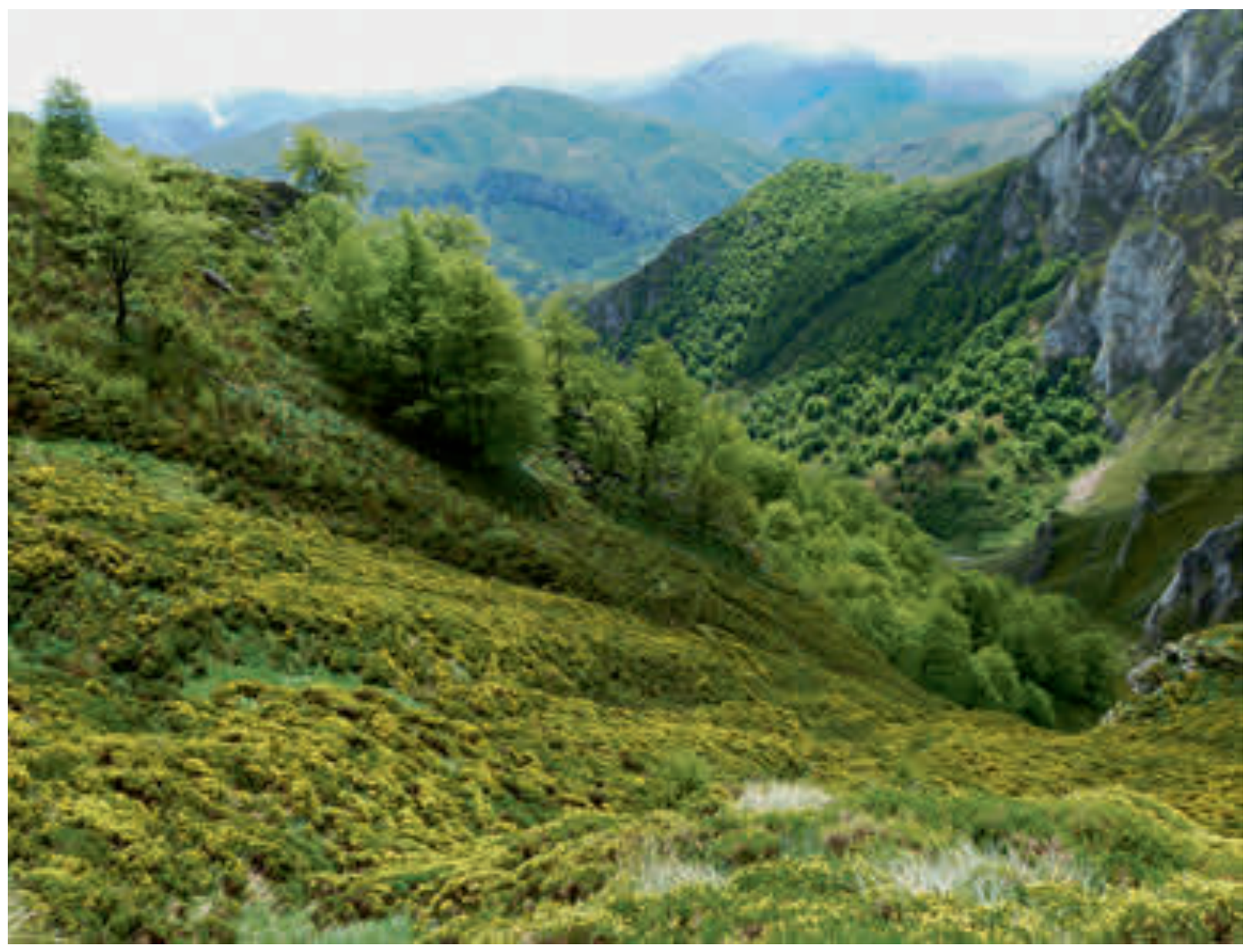

1500 m Höhe gedeiht zudem Narcissus citrinus, ein Endemit des Kantabrischen Gebirges. Die blassgelben bis zitronenfarbigen Blüten dieser kleinen Narzissen-Art haben eine $2-3 \mathrm{~cm}$ lange Nebenkrone.

Auf dem feuchten Waldboden leben viele kleinere Tiere. Immer wieder kann man zahlreiche Spanische bzw. Große Nacktschnecken (Arion vulgaris) beobachten. Die ursprünglich nur auf der Iberischen Halbinsel und in WestFrankreich heimische Art wurde in Mitteleuropa eingeschleppt, wo sie auf landwirtschaftlichen Flächen großen Schaden anrichten kann.

Die Picos de Europa sind landwirtschaftlich geprägt. Idyllisch in die Landschaft der collinen und montanen Stufe integriert sind kleine Bergdörfer mit schönen alten Steinhäusern, mitunter sogar Wachtürmen, die noch aus dem Mittelalter stammen (z. B. Redo, Mogrovejo, San Estebano, Cain, Bulnes). Alte traditionelle Wege verbinden manche Dörfer miteinander, dementsprechend führen auch noch einzelne sehr schöne alte Steinbrücken über die reißenden Bergflüsse. Die Menschen leben von Landwirtschaft und Tourismus. Die Dörfer sind von Mähwiesen und Weiden umgeben. Im Sommer werden die Rinder auch auf die höher gelegenen Almwiesen getrieben. Die Tiere werden zur Fleischgewinnung und vor allem für die Milchproduktion gehalten. Ein großer Teil der Milch wird zu Käse verarbeitet, dementsprechend viele Käsereien gibt es. Berühmt ist der Blauschimmelkäse von den Picos de Europa. An das raue Klima in den kantabrischen Bergen angepasst ist die spanische Hausrinderrasse Tudanca, die in einigen Merkmalen dem ausgerotteten Auerochsen ähnelt. Diese alte Tierrasse ist bedroht und deshalb als schützenswert eingestuft. Durch die kantabrischen Bergbauern wird sie erhalten. Etwas ungewöhnlich wirken auch die Hunde, die in jedem Dorf anzutreffen sind, denn mit ihren langen, lockigen Haaren sehen sie aus wie eine Mischung zwischen Teddybär und Schaf. Es handelt sich um 


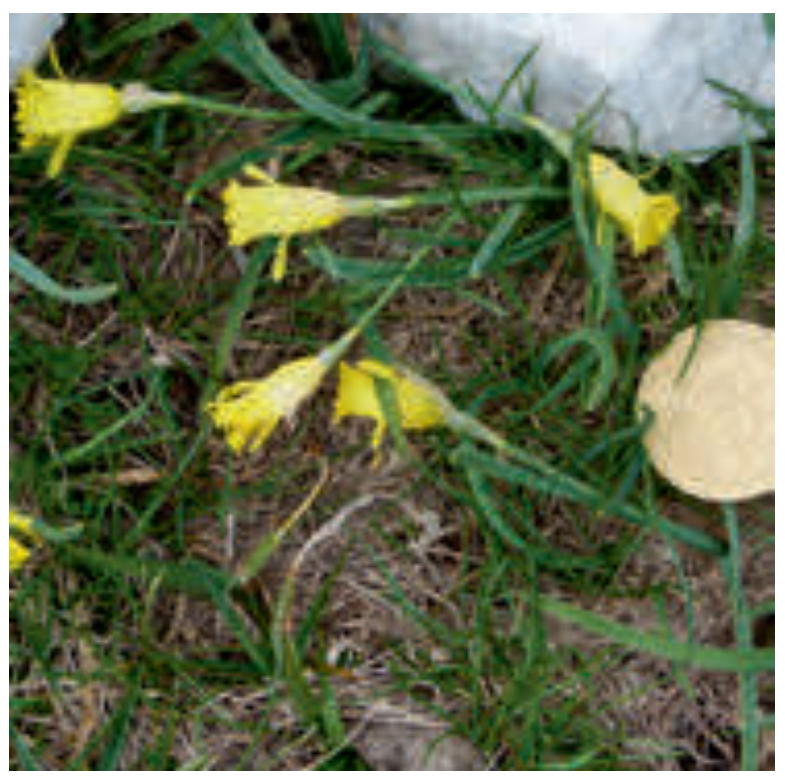

den Spanischen Wollhund (Perro de Agua Español), wobei in Nordspanien vor allem die Form Perro del Cantabrico als Haushund sowie zum Hüten von Schafen gehalten wird.

\subsection{Subalpine Stufe}

Nach oben schließt sich bis auf $2200 \mathrm{~m}$ die subalpine Stufe an. Bäume fehlen hier nun. Es können sich nur noch kleinere Gehölze wie z. B. der Zwerg-Wacholder (Juniperus nana) halten. Ein Endemit West-Europas ist das auch tiefer in den Wäldern vorkommende Heidekrautgewächs Daboecia cantabrica mit seinen leuchtend pink gefärbten Blütenglöckchen. Der Gattungsname bezieht sich auf DABEOC vON Lough Derg, einem irischen Heiligen. Verbreitet ist $D$. cantabrica außer in Nordspanien auch in Irland und auf den Azoren. Es handelt sich um eine Charakterart der Atlantischen Heiden. Da die Almwiesen stark beweidet werden, halten sich hier vor allem solche Arten, die stark bewehrt oder giftig sind. So gibt es Wiesen mit uns aus den Alpen vertrauten Trollblumen oder mit Weißem Affodill (Asphodelus albus). Äußerst stechend ist das Pyrenäen-Männertreu (Eryngium bourgatii, Spanische Edeldistel). Aufgrund seiner attraktiven stahlblau gefärbten köpfchenförmigen Dolden findet man es auch als Zierpflanze in unseren Gärten.

Im Mai sind die Berge besonders attraktiv, da sie stellenweise flächendeckend gelb gefärbt

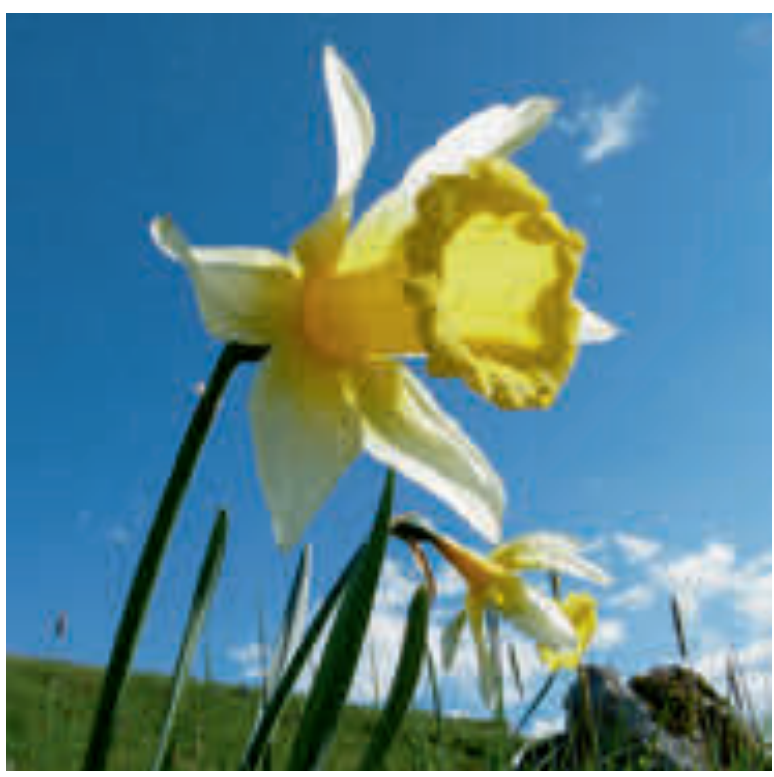

sind, wenn der stark stechende Spanische Ginster (Genista hispanica subsp. occidentalis) blüht. Weitere Ginster, die in dieser Zeit ebenfalls blühen, sind Genista legionensis, Genista obtusiramea und der dem Besen-Ginster sehr ähnliche Cytisus cantabricus. Unter den Orchideen sind besonders häufig Händelwurz (Gymnadenia conopsea), Pyramiden-Orchis (Anacamptis pyramidalis), Kleines Knabenkraut (Orchis morio) und Ständelwurz (Serapias lingua, S. vomeracea). Sehr lohnenswert sind die gen Süden ausgerichteten Bergwiesen am Pass Puerto de Piedrasluengas. Ende Mai blühen dort noch in großer Anzahl die nur im Kantabrischen Gebirge heimischen Spanischen Narzissen (Narcissus nobilis var. leonensis $=$ Narcissus pseudonarcissus subsp. leonensis). Im Gegensatz zur "normalen“ Narcissus peudonarcissus sind die besonders attraktiven Blüten größer und kräftiger. Mit ihr vergesellschaftet sind z. B. der Stängelumfassende Hahnenfuß (Ranunulus amplexicaulis), ein Endemit der Berge Nordspaniens oder das Hornveilchen (Viola cornuta), aus dem die bei

Abb. 12 (links): Narcissus nobilis var. leonensis.

Abb. 13 (rechts): Narcissus asturiensis.

Abb. 14 (Seite 103 oben): Cares-Schlucht, links im Bild der künstlich angelegte Wasserkanal.

Abb. 15 (Seite 103 unten): Ende Mai kann auf den höchsten Gipfeln noch viel Schnee liegen. 
uns beliebten Balkonpflanzen hervorgegangen sind. An besonders feuchten Stellen gedeiht Pinguicula grandiflora, eine carnivore Art mit im Vergleich zu den alpinen Arten relativ großen Blüten.

Auf steinigem Untergrund wächst häufig das Drachenmaul (Horminum pyrenaicum), das auch in den Alpen weit verbreitet ist und dort auf über $3000 \mathrm{~m}$ Höhe steigt. Sehr häufig sind auch Steinsame (Lithodora diffusa) und Leberbalsam (Erinus alpinus). In Felsritzen, oft aber auch in Mauerfugen, findet man die großen, kugeligen Polster von Saxifraga canaliculata. Wie winzige Zapfen wirken die Triebenden von Saxifraga conifera, einem Endemiten des Kantabrischen Gebirges, der von etwa $1200 \mathrm{~m}$ bis auf rund $2500 \mathrm{~m}$ Höhe steigt (Nava \& Fernandez CASAdo 1995)

\subsection{Alpine Stufe}

In der obersten alpinen Stufe halten sich nur noch diejenigen Arten, die starken Wind, hohe Sonneneinstrahlung, niedrige Temperaturen
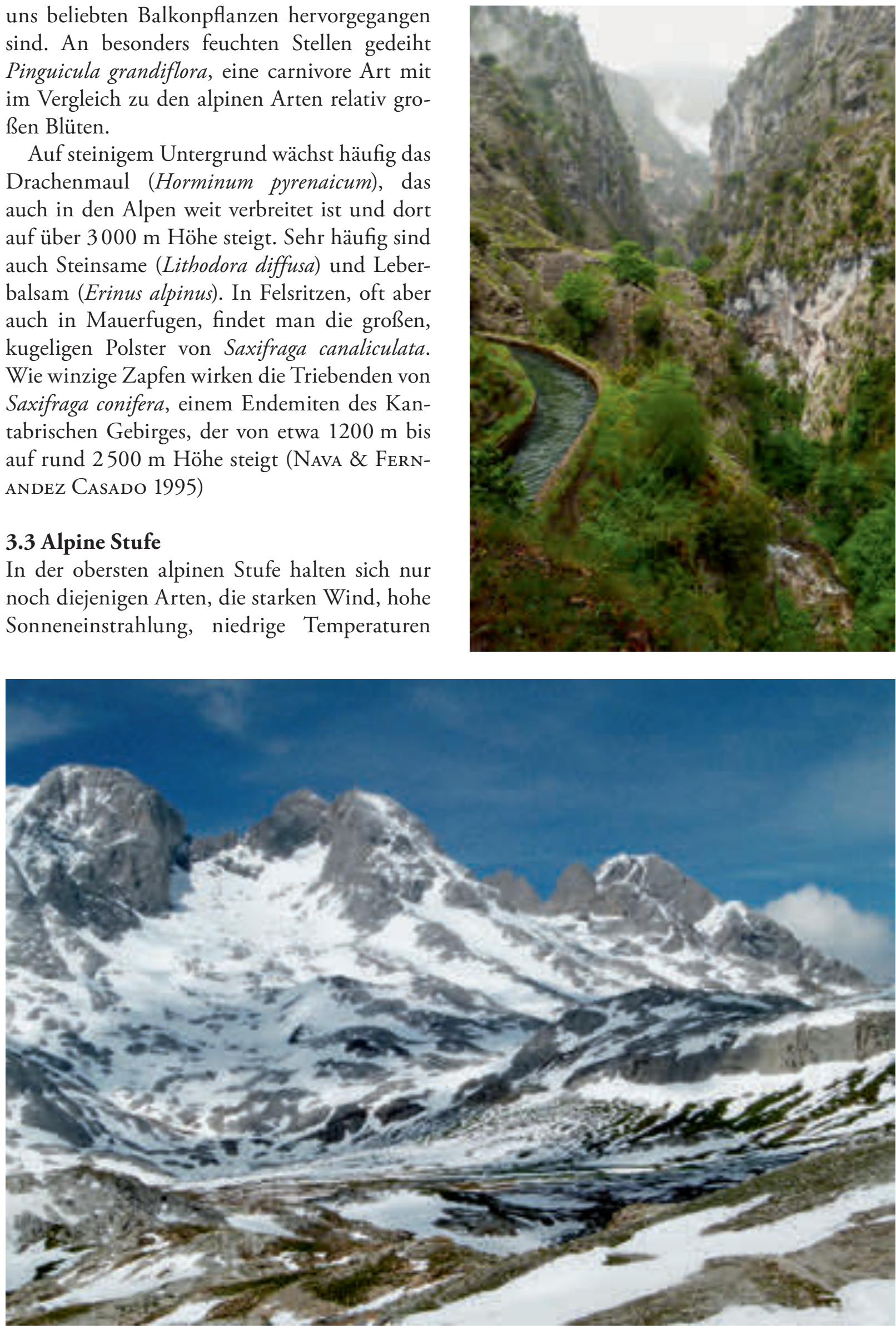

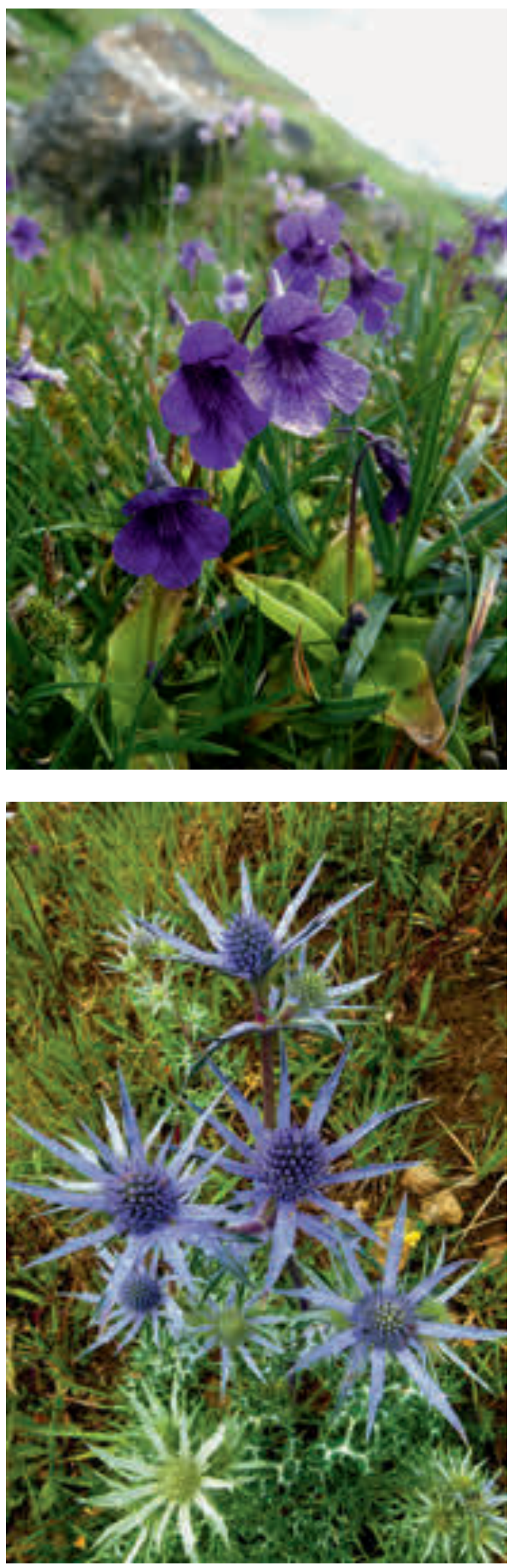

und lange Schneebedeckung ertragen können. Typische Vertreter sind Enzian, Kugelblume, Mannsschild oder Leinkraut, hier vertreten durch die Arten Gentiana angustifolia subsp. occidentalis, Globularia nana, Androsace villosa und Linaria alpina subsp. filicaulis. Zwischen Kalkgeröll hält sich ein kleines Löwenmäulchen (Chaenorrinum origanifolium). In den höheren Lagen und auch etwas niedriger auf Wiesen (bevorzugt auf öberflächlich versauertem Boden) wächst die kleinste Narzisse der Welt (Narcissus asturiensis $=$ N. minimus). Wenn kurz nach der Schneeschmelze die an Miniaturausgaben von Osterglocken erinnernden Blüten erscheinen, sind die Pflanzen nur wenige Zentimeter hoch. Die Blüten liegen fast auf dem Boden auf. Während der Fruchtreife verlängert sich der Schaft auf rund $15 \mathrm{~cm}$. Die Art wird manchmal auch bei uns kultiviert, gehört dann zu den im Februar am frühesten im Jahr blühenden Arten. In Gartenkultur werden die Pflanzen größer als am Naturstandort.

\section{Schluchten}

Die vielen wilden, tiefen Täler und Schluchten erstrecknen sich oft über mehrere Höhenstufen. Wer von der Küste über Potes nach Fuente Dé zur Seilbahnstation fährt, passiert unweigerlich die Devaschlucht (Desfiladero de la Hermida). Die Straße verläuft entlang des Deva-Flusses in der engen, tief eingeschnittenen Schlucht, die durch schroffe Kalkfelsen begrenzt wird. Besonders die vom Deva-Tal ausgehenden Seitenschluchten (z. B. am Rio Urdon) lohnen sich aus landschaftlicher und botanischer Sicht. Idyllisch in die Landschaft eingebettet sind hier z.B. die Bergdörfer San Esteban, Beges oder Cicera, die jeweils auch gute Ausgangspunkte für Wanderungen sind. Neben Rindern befinden sich hier auch reichlich Pferde und Schafe auf den Wei-

Abb. 16 (oben): Pinguicula grandiflora.

Abb. 17 (unten): Eryngium bourgatii.

Abb. 18 (Seite 105 oben): Leberbalsam (Erinus alpinus) ist eine häufige Art auf steinigen Standorten.

Abb. 19 (Seite 105 unten): Pyrenäen-Gamander (Teucrium pyrenaicum). 

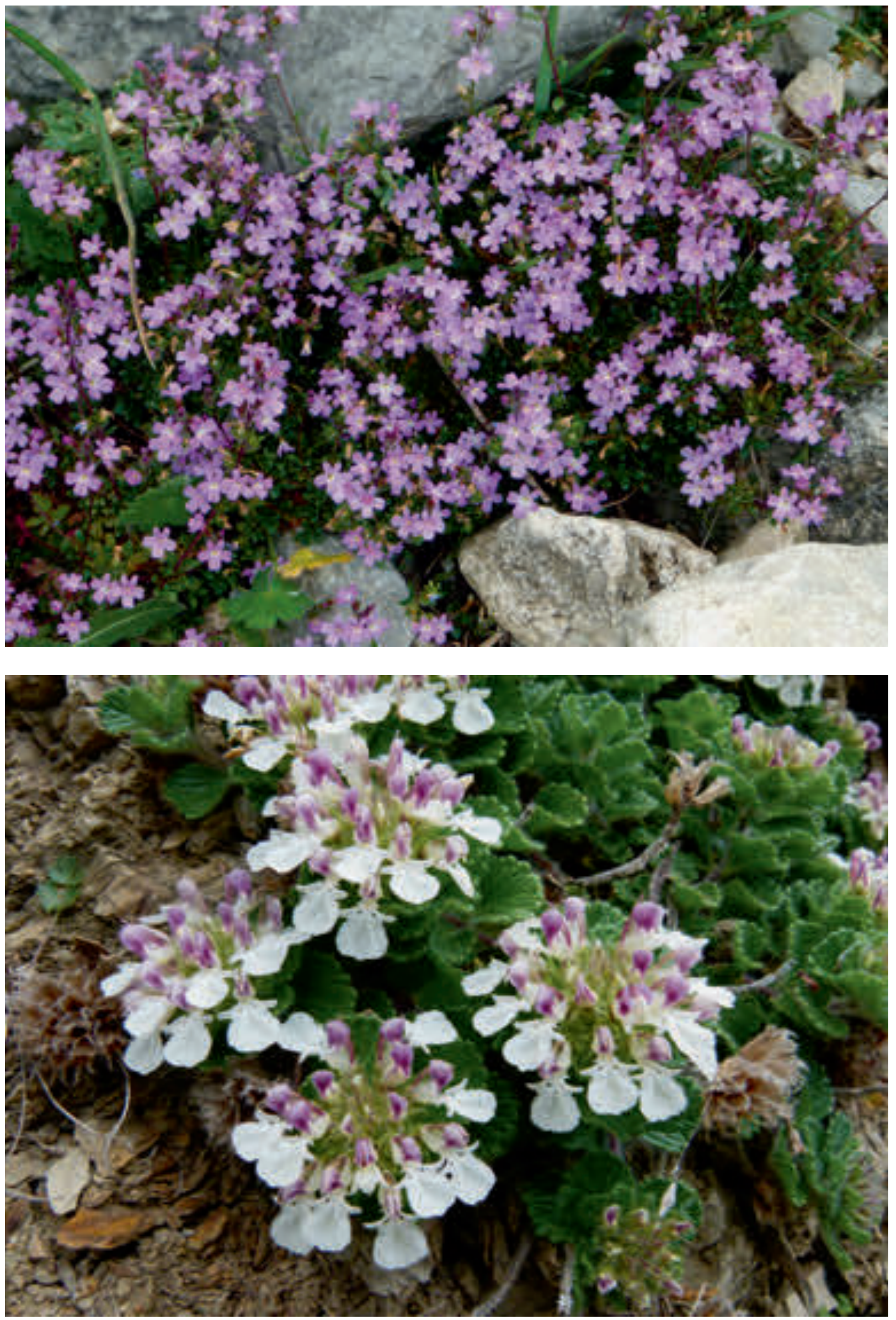

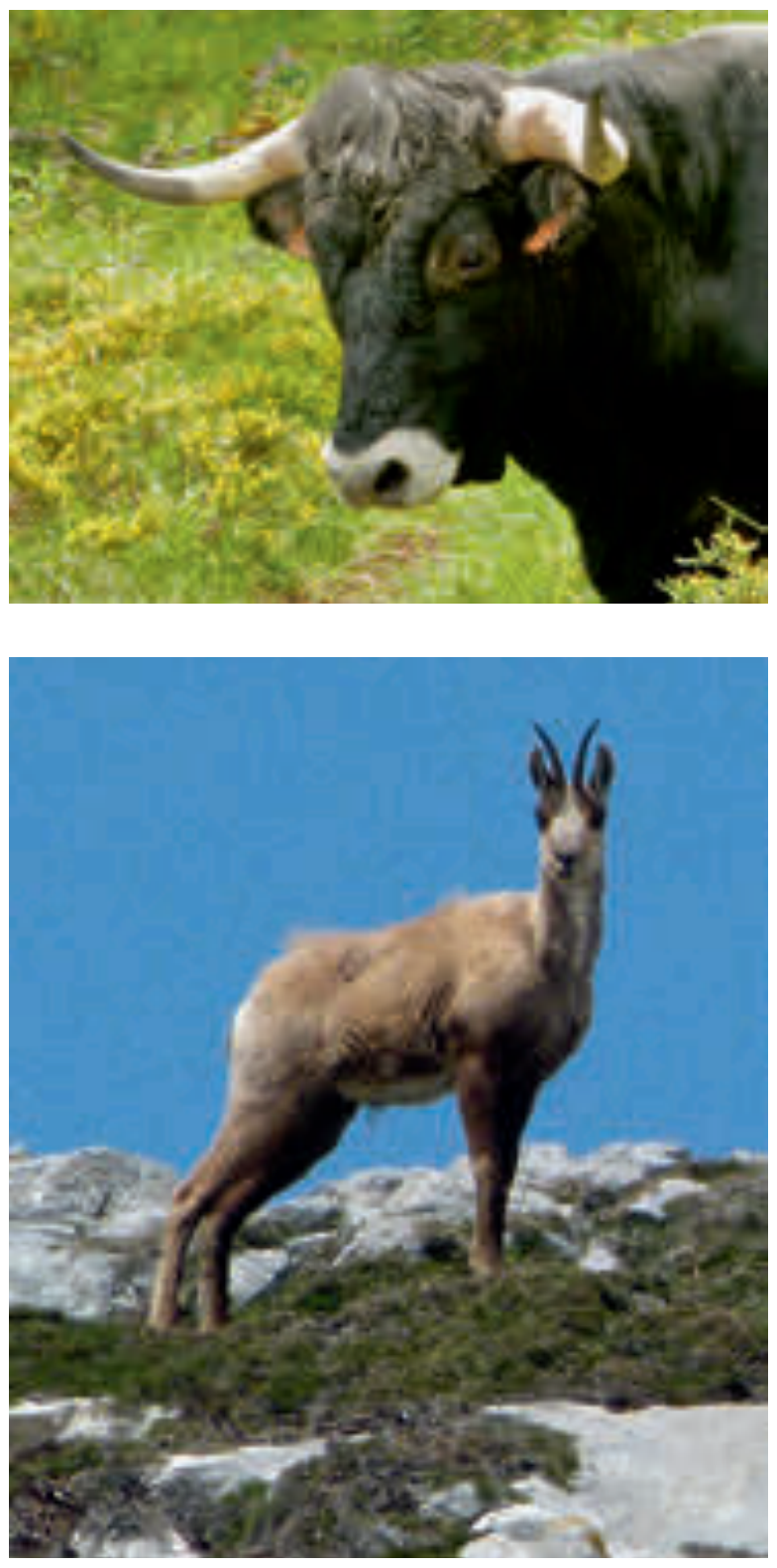

den. An warmen Hängen blühen im Mai verschiedene Orchideen, neben den zuvor genannten auch der Hängende Mensch (Aceras anthropophorum) und das Brand-Knabenkraut (Orchis ustulata). Größere Bestände bildet auf Wiesen der Asturische Klappertopf (Rhinanthus serotinus subsp. asturicus). An besonders feuchten, moorigen Stellen gibt es Wollgras-Arten (Eriophorum) und Läusekraut (Pedicularis mixta).

An warmen, sonnigen Hängen, auch in Felsschluchten, fühlen sich viele Insekten wohl, darunter der seltene Loreley-Dickkopffalter (Carcharodus lavatherae). Der Schmetterling kommt von Nordafrika über Südeuropa bis nach Vorderasien vor mit einer Verbreitungsinsel zwi- schen Lorch und Sankt Goarshausen nahe der Loreley, daher der deutsche Name.

Mit einer Tiefe von $1500 \mathrm{~m}$ ist die CaresSchlucht (Garganta del Cares) extrem gewaltig. Auf einem 1916-1921 in die Felsen gehauenen Fußweg entlang eines zur Stromgewinnung angelegten Wasserkanals ist die Schlucht gut erwanderbar. Mit jährlich rund 300000 Wanderern ist die Cares-Route einer der beliebtesten und meist frequentierten Wanderwege ganz Spaniens. Der Weg verbindet die Dörfer Poncebos in Austurien und Caín in León. Von oben ergeben sich eindrucksvolle Blicke auf die Schlucht, die eine tiefe Schneise zwischen dem Ost- und dem Westmassiv der Picos de Europa bildet. Am Grunde der Schlucht gedeiht die Bruchweide (Salix fragilis). Neben Buchen fühlen sich hier Schluchtwaldarten wie SommerLinde (Tilia cordata) und Esche (Fraxinus excelsior) sehr wohl. Am strömenden Wasser leben Eisvogel und Wasseramsel, aber auch die Nordiberische Kreuzotter.

Wer also eine urspüngliche, wilde Berglandschaft mit bäuerlicher Kultur und spannender Vegetation kennenlernen möchte, ist in den Picos de Europa gut aufgehoben. Wegen des feuchten atlantischen Klimas sind hier die Wälder mit ihren dicken, alten Bäumen, aber auch die schroffe alpine Landschaft in unmittelbarer Nähe zum Atlantik äußerst beeindruckend.

\section{Literatur}

Nava, H. S. \& Fernández Casado, M. A. 1995: Picos de Europa. Flora de alta montana. - Oviedo.

Miguel Menéndez de la Hoz (Red.) o.J.: Parque Nacional de los picos des Europa. Guia de visita. - Oviedo. (auch im Internet unter http://www.magrama.gob.es/ imagenes/es/guia-picos_tcm7-288896.pdf )

\section{Internetseiten}

http://de.wikipedia.org/wiki/Picos_de_Europa

http://de.wikipedia.org/wiki/Nationalpark_Picos_de_ Europa

http://www.topwalks.net/plants/index.htm (Bildergalerie der wichtigsten Pflanzen Spaniens, nach Farben oder Familien sortiert)

Abb. 20 (oben): Tudanca-Rind.

Abb. 21 (unten): Pyrenäen-Gämse. 